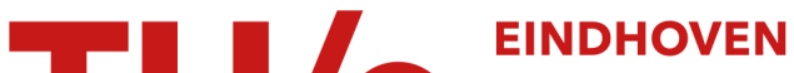 \\ UNIVERSITY OF \\ TECHNOLOGY
}

\section{Cardiac image segmentation for contrast agent videodensitometry}

Citation for published version (APA):

Mischi, M., Kalker, A. A. C. M., \& Korsten, H. H. M. (2005). Cardiac image segmentation for contrast agent videodensitometry. IEEE Transactions on Biomedical Engineering, 52(2), 277-286.

https://doi.org/10.1109/TBME.2004.840500

DOI:

10.1109/TBME.2004.840500

Document status and date:

Published: 01/01/2005

\section{Document Version:}

Publisher's PDF, also known as Version of Record (includes final page, issue and volume numbers)

\section{Please check the document version of this publication:}

- A submitted manuscript is the version of the article upon submission and before peer-review. There can be important differences between the submitted version and the official published version of record. People interested in the research are advised to contact the author for the final version of the publication, or visit the $\mathrm{DOI}$ to the publisher's website.

- The final author version and the galley proof are versions of the publication after peer review.

- The final published version features the final layout of the paper including the volume, issue and page numbers.

Link to publication

\section{General rights}

Copyright and moral rights for the publications made accessible in the public portal are retained by the authors and/or other copyright owners and it is a condition of accessing publications that users recognise and abide by the legal requirements associated with these rights.

- Users may download and print one copy of any publication from the public portal for the purpose of private study or research.

- You may not further distribute the material or use it for any profit-making activity or commercial gain

- You may freely distribute the URL identifying the publication in the public portal.

If the publication is distributed under the terms of Article $25 \mathrm{fa}$ of the Dutch Copyright Act, indicated by the "Taverne" license above, please follow below link for the End User Agreement:

www.tue.nl/taverne

Take down policy

If you believe that this document breaches copyright please contact us at:

openaccess@tue.nl

providing details and we will investigate your claim. 


\title{
Cardiac Image Segmentation for Contrast Agent Videodensitometry
}

\author{
Massimo Mischi*, Member, IEEE, Antonius A. C. M. Kalker, Fellow, IEEE, and Henricus H. M. Korsten
}

\begin{abstract}
Indicator dilution techniques are widely used in the intensive care unit and operating room for cardiac parameter measurements. However, the invasiveness of current techniques represents a limitation for their clinical use. The development of stable ultrasound contrast agents allows new applications of the indicator dilution method. Ultrasound contrast agent dilutions permit an echographic noninvasive measurement of cardiac output, ejection fraction, and blood volumes. The indicator dilution curves are measured by videodensitometry of specific regions of interest and processed for the cardiac parameter assessment. Therefore, the major indicator dilution imaging issue is the detection of proper contrast videodensitometry regions that maximize the signal-to-noise ratio of the measured indicator dilution curves. This paper presents an automatic contour detection algorithm for indicator dilution videodensitometry. The algorithm consists of a radial filter combined with an outlier correction. It maximizes the region of interest by excluding cardiac structures that act as interference to the videodensitometric analysis. It is fast, projection independent, and allows the simultaneous detection of multiple contours in real time. The system is compared to manual contour definition on both echographic and magnetic resonance images.
\end{abstract}

Index Terms-Contrast agents, edge detection, image segmentation, indicator dilution curve, videodensitometry.

\section{INTRODUCTION}

$\mathbf{T}$ HE indicator dilution theory allows the measurement of cardiovascular parameters by analysis of an indicator dilution curve (IDC), i.e., the indicator concentration-versus-time curve. Many techniques are clinically available. They are based on the injection into a vein (either central or peripheral) of an indicator bolus (tracer), such as cold saline (thermodilution), indocyanine green (dye dilution), and lithium [1]-[8]. The injected bolus is detected after the venous returns have been mixed in the right ventricle to build the IDC. Fig. 1 shows an example of lithium IDC. Limitation of current techniques is their invasiveness, since either blood samples are drown (dye and lithium dilution) or a sensing catheter is inserted through the right side of the heart (Swan-Ganz catheter for thermodilution).

The IDC contains all the necessary information for the determination of cardiac output (CO) (blood flow through the heart) and ejection fraction (EF) (percent variation of the ventricular volume) [1], [2], [9], [10]. Also, the pulmonary blood volume

Manuscript received November 10, 2003; revised June 13, 2004. Asterisk indicates corresponding author.

*M. Mischi is with the Eindhoven University of Technology, Eindhoven 5641 GP, The Netherlands (e-mail: m.mischi@tue.nl).

A. A. C. M. Kalker is with the Eindhoven University of Technology, Eindhoven 5641 GP, The Netherlands.

H. H. M. Korsten is with the Eindhoven University of Technology, Eindhoven 5641 GP, The Netherlands and also with the Catharina Hospital, Eindhoven 5641 GP, The Netherlands.

Digital Object Identifier 10.1109/TBME.2004.840500

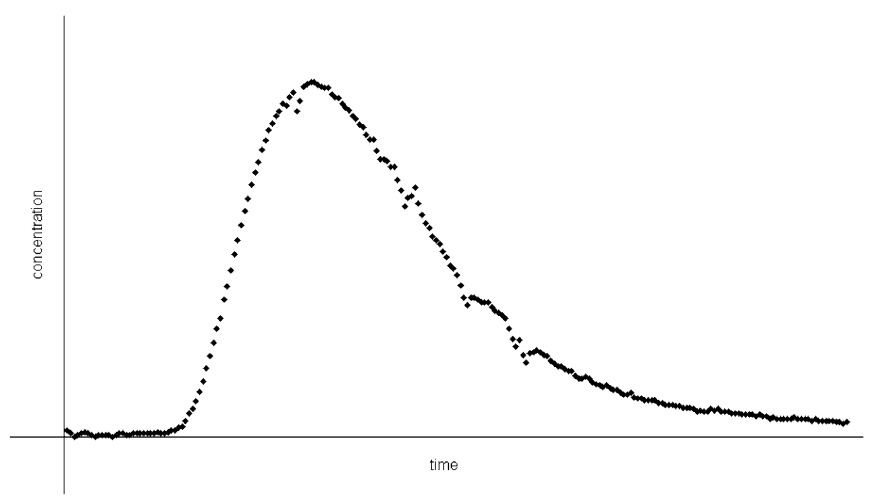

Fig. 1. In vitro lithium IDC.

(PBV) ( blood volume between the pulmonary artery and the left atrium) can be assessed by means of dilution techniques. ${ }^{1}$ It is measured by trans-pulmonary dilution [11]-[13]. A catheter for thermodilution (or dye dilution) is inserted through the femoral artery up to the aorta, where the IDC is measured. Moreover, since the indicator must be injected into the pulmonary artery, a second catheter is needed to reach the injection site. The PBV is determined as the multiplication between the mean transit time (MTT) of the indicator (average opacification delay between the injection and the detection sites) and the CO.

Nowadays, the development of stable ultrasound contrast agents (UCAs) allows a noninvasive application of the indicator dilution method. UCA are micro-bubbles of inert gas stabilized by a shell of biocompatible material (see Appendix A) that are easily detectable by ultrasound analysis [14]-[20].

The B-mode [21] video intensity of the ultrasound scanner increases where UCA bubbles are detected. Since the video intensity is correlated to the UCA concentration [22], [23], an UCA IDC can be determined by videodensitometry of a region of interest (ROI). In vitro studies show that the IDC analysis can be performed with an ultrasound scanner after the relationship between contrast concentration and video intensity (calibration) has been established [22]-[35].

Due to the UCA loss through the lungs, CO measurements must be performed in the right atrium (RA) or right ventricle $(\mathrm{RV})$. This prevents from loosing the relation between injected dose and detected concentration, which is necessary for the $\mathrm{CO}$ derivation [23]. The assessment of RV or left ventricle (LV) EF needs RV or LV videodensitometry, respectively. If the indicator is detected both in the RV and the left atrium (LA), the MTT between RV and LA can be estimated from the measured IDCs and the PBV derived as the product between MTT

\footnotetext{
${ }^{1}$ Actually, standard dilution techniques can only measure the central blood volume, which also includes the average blood volume in the left side of the heart.
} 
and $\mathrm{CO}$ [24]. The implementation of a complete system for in vivo analysis of UCA dilution curves is in progress, but preliminary results concerning EF and PBV measurements are already promising [24]-[36]. An important issue such as attenuation [22] is overcome by injecting a very low dose of contrast [23]. Low signal-to-noise ratios (SNRs) are solved by sophisticated modeling and fitting of the IDC [23], [24].

Although all the studies make use of simple (usually rectangular) manually defined ROIs for UCA IDC measurements [23]-[25], [27]-[30], [33], [34], a crucial issue in the video indicator dilution context for cardiac measurements is the automatic definition of one or more ROIs that stay inside the cardiac walls and maximize the covered surface. Therefore, the contribution of this article concerns the automatic determination of multiple ROIs in real time for IDC measurements. An extremely accurate contour detection is not strictly necessary, but the segmentation must be robust to small SNR and ensure that no tissue is included into the ROI. Signal that is backscattered by the myocardium represents a disturb to the IDC measurement and should not be included in the ROI. A manual delineation of the ROI does not ensure to maximize the measurement area and slows down the operating room practice. The expansion of the ROI leads to larger average and IDC noise reduction. As a consequence, the interpretation of the IDC is more accurate.

Required features for a suitable image segmentation system also include a simple and efficient interface to the user in order to allow operating room applications. The adaptability to different echo-cardiographic probes (trans-esophageal and transthoracic ${ }^{2}$ [37]), views, and contour shapes is necessary to use the system for different measurements (e.g., CO, LV and RV EF, PBV, etc.) and pathologies (e.g., an aneurysm). Moreover, the simultaneous detection of several contours in real time should be achieved without the employment of expensive hardware.

Several techniques are reported in literature for contour detection. However, it is difficult to determine a general solution that is optimal in terms of accuracy, reliability, and computation time. The most common approaches for cardiac-chamber contour detection can be divided into five main groups [38]-[44]: two-dimensional (2-D) filters, morphologic segmentations, model-based approaches, active contours, and level set methods.

Two-dimensional filters include all the linear and nonlinear filters typically used for image processing [39], [38]. Different cascade of high-pass filters have been tested, but the robustness of this simple approach with echographic images is not sufficient. The detected edges are fragmented and difficult to interpret. Special algorithms can recombine the edge fragments [45], but they are slow and not applicable for real time processing. Furthermore, false edges due to speckle noise ${ }^{3}$ [21], [38] should be distinguished.

Morphologic segmentations [46], [38] apply a threshold to gray-level images and transform them into binary ones, which are processed by binary filters. This approach seems to be not

\footnotetext{
${ }^{2}$ In trans-esophageal echo-cardiography, the ultrasound transducer is inserted through the mouth into the esophagus, very close to the heart, while in transthoracic echo-cardiography, the probe is external, positioned on the chest.

${ }^{3}$ Speckle is the typical texture that characterizes echographic images. It is the result of echoes generated by small scatterers randomly distributed in tissues. Scattering effects that are generated outside the main lobe of the ultrasound beam also contribute to generate speckle noise.
}

appropriate for contour detection in echographic images. The gray-levels that define the interface blood-myocardium is not a stable and reliable reference. As a consequence, the binary images can show several connections between different chambers or even segmentation of the chamber surface into separate particles. The selection of an appropriate threshold to generate the binary image is a critical point since it is difficult to define an algorithm that suits all the images [41]. A cascade of morphologic filters such as erosion and dilatation can solve some of the problems, but requires a large increase of computations.

Model-based approaches, such as active appearance models [42]-[44], [47]-[49] first applied for face recognition, are based on principal components analysis of a set of training images in order to build a mean shape model. An eigenvalue problem is solved to best adapt the model to the detected contours. When the mean shape model can represent the chamber contour, this method shows a reliable behavior. However, unless some extraconstraints are defined [50], the model fitting requires the optimization of a large set of parameters. One more disadvantage of model-based algorithms is their limitation to a specific contour shape. Different projections, such as long and short axis views, ${ }^{4}$ require different models.

Active contour model or Snake techniques minimize an energy function associated to the contour curve (usually represented by a B-Spline) [39], [40], [51]. The total energy is defined as the sum of an internal energy and an external energy. The internal energy depends on the characteristic of the spline curve (elasticity and stiffness), while the external energy depends on the matching between the spline curve and the image features (gray level value, gradient, etc.). Since they are iterative techniques that locally deform the snake (spline), the user must define an initial contour (set of points). Each point of the initial contour is iteratively adjusted until the total energy of the spline, which interpolates all the points, reaches a minimum. The final result depends on the initial conditions and the weights associated to the internal and external energies. Unfortunately, the solution can easily converge into local minima. Moreover, especially with echographic images, the snake is easily attracted by features that are due to noise, ending with wrong results. A smoothing prefilter can partially prevent from these errors, but reduces the gray-level gradient along the contours.

The level set method [52], which more in general can be considered as an optical-flow approach, is an emerging technique for tracking moving interfaces. The combination of segmentation and tracking allows exploiting the additive information that derives from motion. The algorithm uses a deformable contour that moves using gradient descent and seeks for local solutions. As a consequence, a powerful initialization technique is required (as for the snake technique). In fact, the final contour is strongly dependent on its initial position. The function that defines the deformable contour model allows the representation of any shape. The time differentiation of the contour function leads to a Hamilton-Jacobi type equation. The solution of this equation provides the contour segmentation. The final solution is a compromise between attraction to image features (e.g., the gradient) and contour smoothness. Computational cost, initialization, and noise sensitivity issues should be carefully considered.

\footnotetext{
${ }^{4}$ The long and short axis views are the longitudinal and transverse plane projections of the ventricles, respectively.
} 
For all the mentioned methods, the initialization is a critical issue and can be hardly automated. An initial manual definition of a few reference points or the entire contour is usually needed. The result is a difficult application of the system and the need for the employment of trained expertises. As a consequence, these algorithms do not suit the requirements for emergency routines and operating room applications. Moreover, the available algorithms are either insufficiently accurate, or constrained into a specific shape (or model), or computationally too expensive for multiple simultaneous contour detections in real time [44]. Therefore, in this specific context, none of the previously mentioned algorithms seems to fit the required characteristics.

This article presents a new automatic cardiac-wall tracking (contour-detection) algorithm dedicated to UCA IDC analysis. The IDCs are measured simultaneously in different cardiac cavities (ROIs), so that the measurement of various cardiac parameters, such as CO, EF ( $\mathrm{LV}$ and RV), and PBV, is feasible by a single UCA bolus injection.

The computational cost of the implemented algorithm is limited, so that real-time detection of multiple ROIs does not require expensive hardware. Furthermore, the contour detection is projection-independent and allows the use of any cardiac view to the cardiologist.

The input of the system is the B-mode video output of the ultrasound scanner, which is processed in real time. Only a simple mouse click in the center of the cavities of interest is required by the user, resulting in a system that is also suitable for operating room applications. An off-line analysis of stored digital (AVIs) or analog (videotapes) movies is also integrated in the current set-up.

For IDC measurements the ultrasound scanner is set on fundamental mode (i.e., the transducer transmits and receives at the same frequency ${ }^{5}$ ) in order to have an easier calibration of the system. The segmentation algorithm is based on a radial high-pass filter with an automatic outlier suppression. It is general enough to be also adopted for magnetic resonance imaging (MRI) analysis of gadolinium dilutions [55], [56].

The ROI is determined before the chamber opacification to avoid confusion between contrast and tissue. The contour tracking is performed for two cardiac cycles before the contrast injection. The minimum-area contour is automatically determined and used as the ROI for the following IDC measurement. The ROI is fixed inside the cardiac chamber while the contrast is flowing. The mean gray levels in the ROIs are processed in order to obtain the IDCs (videodensitometry). The use of the minimum-area contour over two cardiac cycles ensures that the cardiac walls, which introduce noise (high gray levels) into the measured IDC, are excluded from the ROI during the measurement.

The accuracy of the border detection is validated by comparison with manual contour delineation over a set of 40 ultrasound echo images and 20 MRIs. Furthermore, the complete system is tested with a series of 12 UCA injections to evaluate the IDC fit improvements with respect to manual ROI definition. Accurate contour detections and IDC fits encourage the employment of the system for indicator dilution videodensitometry.

${ }^{5}$ In UCA echography the nonlinear response of the bubbles is often exploited by receiving at higher frequencies (second or higher harmonic) [14], [53]. However, the use of the fundamental mode filters out the nonlinear contributions and increases the linearity of the response.

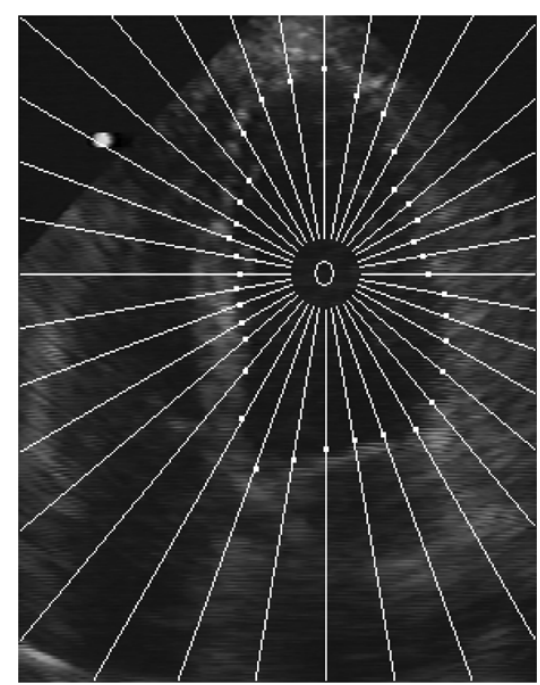

Fig. 2. Beam of rays from inside a left ventricle. The small squares represent the intersection between the rays and the endocardium.

\section{Methodology}

The proposed contour detection algorithm is initially designed for echographic images, which are the most difficult to treat due to their low SNR. However, once echographic images are successfully analyzed, the same algorithm is easily adapted to MRI, since the image contrast and the SNR are much larger.

The echographic B-mode view of a cardiac chamber consists of a dark region (the blood-filled inner of the chamber) surrounded by a bright structure due to the sound that is backscattered from the cardiac-wall-to-blood interface. A chamber can be interpreted as a 2-D convex object. A radial beam of rays that is originated at any point inside the chamber intercepts the border and defines the contour (see Fig. 2). The presented contour detection approach is based on a radial edge-detection filter.

An edge produces spatial high-frequency components and a high-pass filter can be used as an edge detector. Therefore, the basic principle of the proposed contour detection algorithm is the application of a mono-dimensional high-pass filter along a set of rays whose origin is determined by a simple mouse click, which makes the user interface simple and friendly. The origin point of the set of rays should be central, but not precisely in the chamber centroid. The transformation of a bi-dimensional problem into a mono-dimensional problem reduces the computational complexity and allows real-time applications of the system.

A linear high-pass filter is applied along each ray. The impulse response consists of two normalized rectangles, as shown in Fig. 3. The rectangle length and amplitude are equal to $\Delta$ and $\pm 1 / \Delta$, respectively. One rectangle is positive while the other one is negative. The distance between the rectangles is determined by $d(d \geq 0)$. The filter is basically a modified radial-gradient operator. If $I\left(x_{k}, y_{k}\right)$ represents the pixel intensity (gray level) along a radial line, than the discrete radial filter convolution is implemented as given in (1)

$$
\begin{aligned}
\xi_{k}=\frac{1}{\Delta} \cdot \sum_{j=0}^{\Delta-1} I\left(x_{\left(k+j+\left\lceil\frac{d}{2}\right\rceil\right)}, y_{\left(k+j+\left\lceil\frac{d}{2}\right\rceil\right)}\right) \\
\quad-\frac{1}{\Delta} \cdot \sum_{j=0}^{\Delta-1} I\left(x_{\left(k-j-1-\left\lfloor\frac{d}{2}\right\rfloor\right)}, y_{\left(k-j-1-\left\lfloor\frac{d}{2}\right\rfloor\right)}\right) .
\end{aligned}
$$




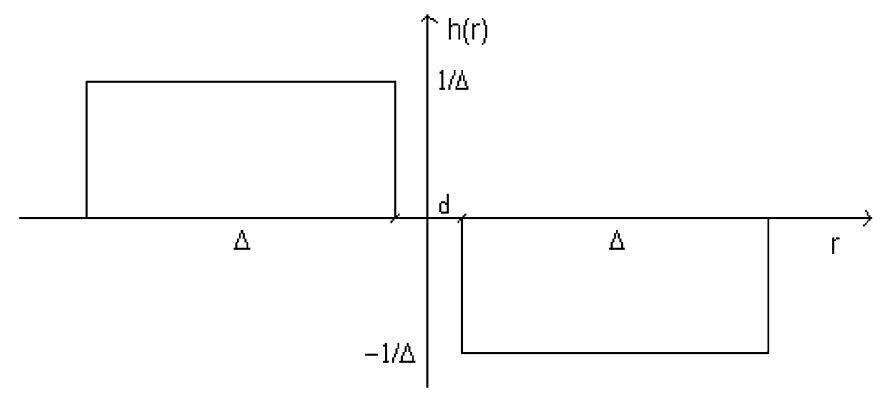

Fig. 3. Impulse response of the radial filter. It is the function that is convoluted with the pixel intensities along each defined radial line. The distance from the radial line origin is represented by the variable $r$. The impulse response is made of two rectangular functions with the same length $\Delta$ and amplitudes equal to $1 / \Delta$ and $-1 / \Delta$. The distance between the rectangles is equal to $d$.

The detected edge point along each ray corresponds to the first location $\left(x_{k}, y_{k}\right)$ where the filter output $\xi_{k}$ surpasses a predefined threshold.

A set of parameters controls the filter, so that the system is adaptable to different image features and imaging techniques. The parameters are the length $\Delta$ and the mutual distance $d$ of the impulse response rectangles, the number of rays, and the threshold. For echographic images we usually fix the number of rays and $d$ to 360 and 2 (pixels), respectively. Typical ROI areas in echographic images (including all possible cardiac views and chambers) go from 5000 to 50000 pixels, therefore, 360 edge points are sufficient to reconstruct the contour. If the contour is very small (perimeter shorter than 500 pixels), then the number of edge points should be decreased. The choice for $d$ is not critical. The length of the rectangle $\Delta$ is fixed to 20 in order to detect the edges and filter out the speckle noise, which consists of spots that cover less than 50 pixels.

The threshold can be adjusted manually. Usually, for well equalized images, a contrast of 20 gray levels leads to the most accurate results. However, an automatic threshold estimation is implemented too. Based on the histogram of the image, the gray level standard deviation $\sigma$ is calculated and the threshold $S$ is determined as given in (2). The logarithm is used to compress the range of $\sigma$, which for echographic images covers a wide interval

$$
S=\lceil a \cdot \ln (\sigma+1)\rceil
$$

The value for the coefficient $a$ is optimized over a set of 40 echographic images and fixed to 6.7. Also the use of the threshold proposed by Otsu has been investigated [41], however, the threshold defined in (2) suits better for this specific application and shows a more stable behavior. In fact, differently from typical morphologic applications, in this context the threshold problem regards the radial gradient rather than the absolute value of the video intensity.

A median filter [38], [39] is applied before the proposed radial filter to remove the spots due to speckle. The choice for a nonlinear filter over a linear low-pass filter is due to the need for removing the speckle without blurring the image. A blurred image makes the subsequent edge detection less efficient. Instead, a median filter enhances the sharpness of continuous contours. The implemented filter is a $5 \times 5$ eighth-order median filter. The pixels covered by the $5 \times 5$ kernel are ordered from

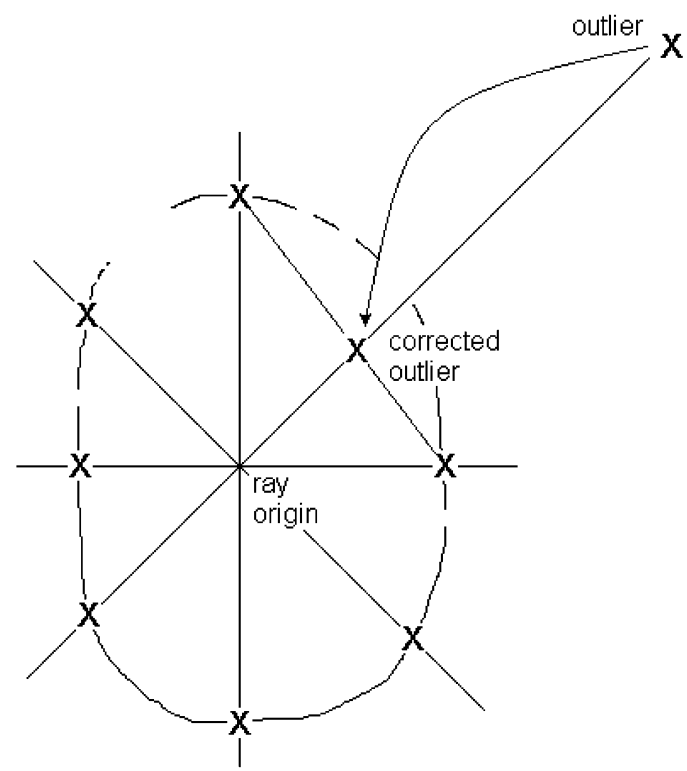

Fig. 4. Schematic example of outlier correction. The outlier is replaced by the intersection between the ray where it lay and the linear-interpolation line of the detected edge points.

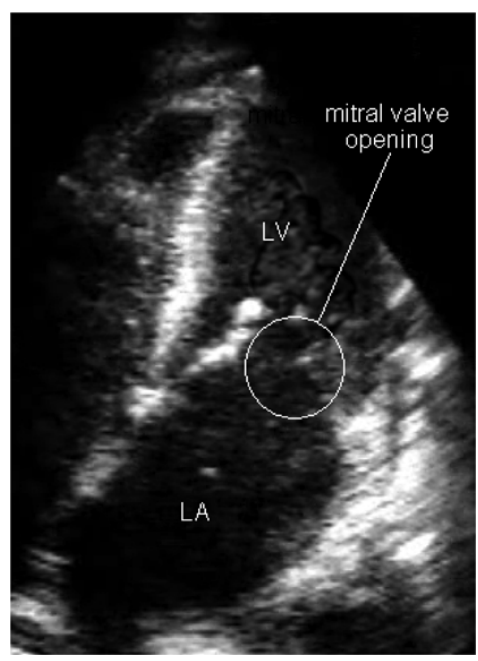

Fig. 5. Two-chamber view with open mitral valve. No ultrasound waves are reflected through the open valve and the resulting echo-image shows the LA and $\mathrm{LV}$ as two connected cavities.

the minimum to the maximum gray-level. The pixel that corresponds to the center of the kernel is then substituted with the 9th pixel in the ordered list (eighth-order median filter). The size is chosen according to the typical size of the speckles.

A linear interpolation of the points that are detected by the radial filter (they should be 360 , one for each degree, but along some rays the edge could be undetectable) defines the ROI. Unfortunately, due to the low quality of echographic images, the ROI contains several outliers (see Figs. 4 and 6), and expands beyond the cardiac walls. In fact, very often, not all the cardiac wall-to-blood interface gives a good ultrasound reflection, and entire parts of the contour may be completely unrecognizable. Moreover, in long-axis projections the cardiac chamber is open through the valve (see Fig. 5).

A routine is implemented to detect and correct the outliers (see Fig. 4). It is based on the assumption of continuous and 


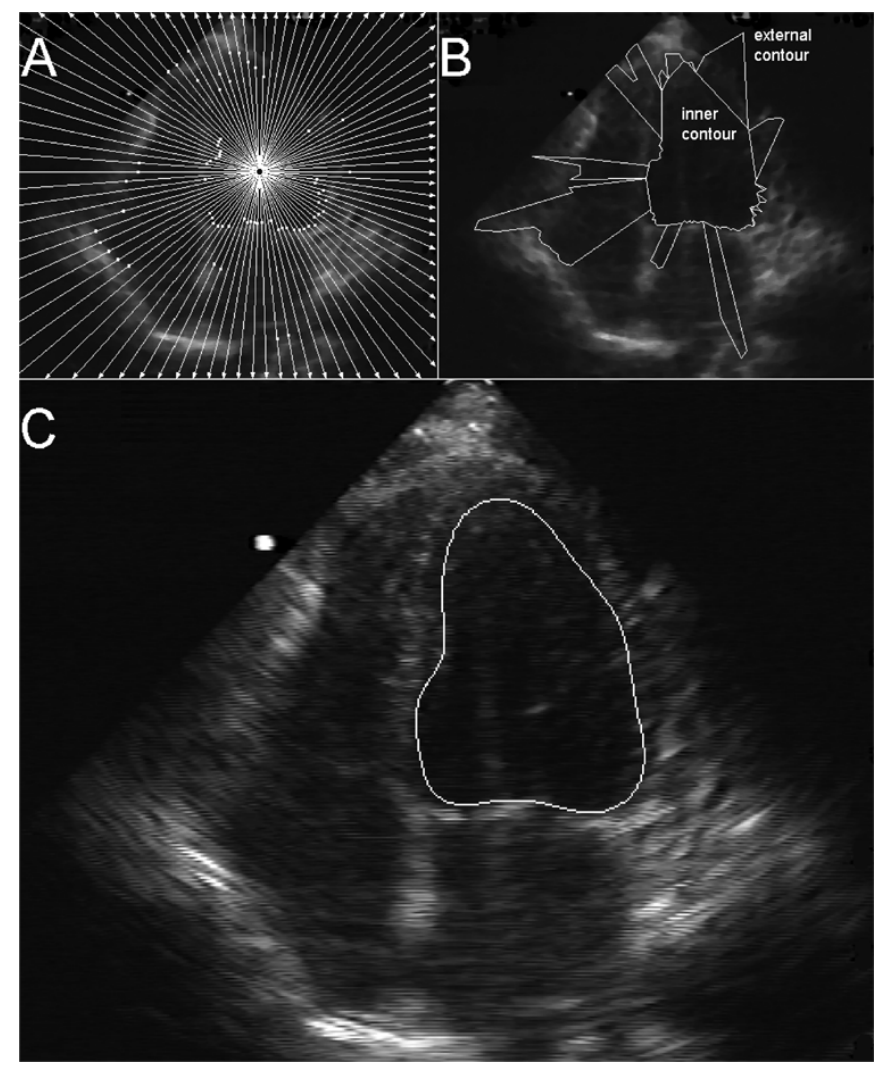

Fig. 6. The upper figures show (A) the high-pass radial filter and (B) the outlier correction. The external contour includes the outliers while in the internal contour they are corrected by derivative filtering. Fig. (C) shows the final smoothed contour.

smooth edges. The Cartesian coordinates of the detected edge points are transformed into polar coordinates centered in the origin of the rays (see Fig.7). This mono-dimensional polar plot (distance from the origin versus angle) is processed to remove the outliers. The first derivative of the plot is calculated and the points whose amplitude surpass a fixed threshold removed.

The hypothesis of bounded derivative is a consequence of the assumption of continuous and smooth edges. After an experimental optimization process, the threshold has been determined as to be equal to $7 \%$ of the standard deviation of the initial polar plot (before the outlier correction). For each removal the derivative function is updated. The process is repeated (clockwise and anticlockwise $\left.{ }^{6}\right)$ until all the points satisfy the threshold condition.

Once the outliers are removed, a new ROI is defined as the linear interpolation of the remaining edge points (see Fig. 4). Therefore, the removed edge points are replaced on the intersection between the interpolation line and the rays where they lay.

Usually the resulting ROI is too sharp (i.e., defined by many sharp angles), especially when several outliers are corrected. A more "anatomic" shape of the ROI is obtained by low-pass filtering (smoothing) the polar plot after the correction of the outliers. Before the contour smoothing, the ROI centroid is calculated and the polar coordinates referred to the new origin. This

${ }^{6}$ The derivative discontinuities may be asymmetric and must be detected in both directions.
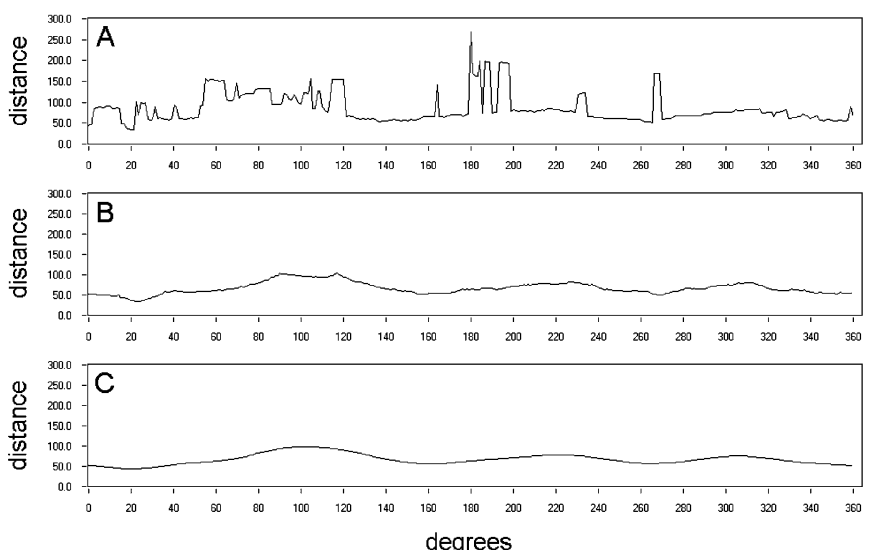

Fig. 7. With reference to Fig. (6), (A) is the polar coordinate representation (distance from the radius origin versus angle) of the edges that are detected by the radial high-pass filter. (B) and (C) show the effect of the outlier correction and smoothing processes, respectively.

operation ensures a better independency of the smoothing filtering from the first origin-point choice.

The adopted filter is a zero-phase low-pass filter, designed as a cascade of a causal FIR (Finite Impulse Response) low-pass filter and a phase shifter. The DC component of the filter impulse response is normalized to a value that is smaller than one, so that the ROI area is slightly decreased. This ensures the cardiac walls to be not included in the ROI. Figs. 6 and 7 show the contour detection process in Cartesian and polar coordinates, respectively.

As presented in previous literature [57] and despite the accurate contour detection that is shown by the proposed method, the addition of an active contour (snake) optimization is tested. The initial contour is defined by the output of the previous algorithm. The algorithm developed by Amini et al. [58] has been modified and adapted to the radial structure of the system. The results for this implementation are not encouraging. The snake optimization process requires a considerable increase in computations while the accuracy improvement is limited. Therefore, we have chosen not to implement the snake optimization into the IDC analysis system. Future research will consider alternative radial active contour optimizations.

Typically, the measurement of an IDC takes more than $20 \mathrm{sec}-$ onds, and during this time the cardiac walls must stay outside the ROI. Because of the cardiac wall motion, a new ROI should be defined for each frame (wall tracking). Unfortunately, the ROI cannot be determined after the contrast appears in the chamber because the backscatter due to the cardiac tissue is confused with that due to the contrast (fundamental mode echo-analysis). Our solution is to determine the ROI before the contrast appearance and to keep it fixed during the IDC measurement. To ensure that the myocardium is never included, the minimum-area ROI is selected during two cardiac cycles before the chamber opacification.

In conclusion, the in vivo IDC measurement is performed in three phases.

- A ROI is defined for each frame (wall tracking) during two cardiac cycles before the chamber opacification.

- The minimum-area ROI is automatically determined and fixed for the videodensitimetry. 


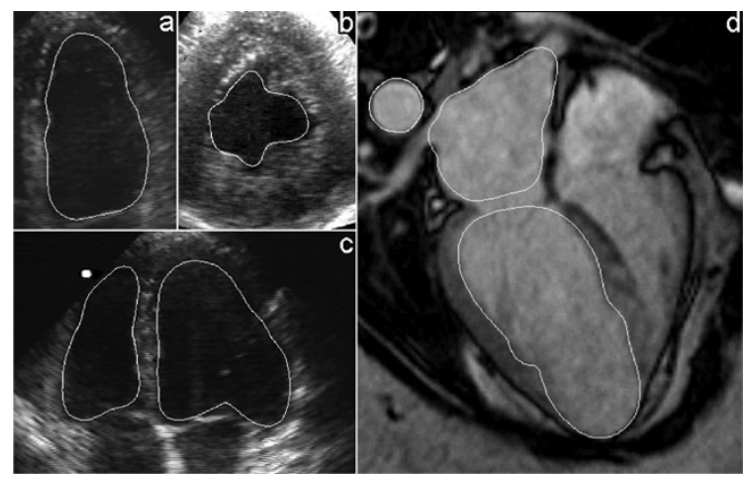

Fig. 8. Image a and $\mathrm{b}$ show the contour detection in a long-axis view and a short-axis view of the left ventricle. Image $\mathrm{c}$ shows a four-chamber view with the detection of the left and right ventricles. Image d shows the detection of different ROIs (left ventricle, left atrium, and aorta) in a MRI frame.

- The mean gray-level is calculated over the fixed ROI while the contrast bolus is flowing.

This algorithm is implemented in software and hardware to establish the performance of the algorithm in a realistic environment. The software implementation is made in Labview ${ }^{\circledR}$, Imaq Vision $^{\circledR}$, and Matlab $^{\circledR}$. The developed software can process AVI files as well as it can be interfaced to the video output of either an ultrasound scanner or a videorecorder. The video interface is realized by the NI 1407-PCI ${ }^{\circledR}$ frame grabber, which is controlled via Labview ${ }^{\circledR}$ and MAX $^{\circledR}$ (Measurement and Automation Explorer). The video grabber can interface both European (CCIR) and American (RS-170) standards [59].

\section{RESULTS}

The proposed contour detection algorithm is applicable to different echographic and MRI cardiac projections as shown in Fig. 8. Since an absolute reference for the contour detection evaluation does not exist, medical image segmentation algorithms are usually compared to manual contour delineation, which is performed by expertises. A set of forty echographic images and 20 magnetic resonance (MR) images is randomly selected from clinical routine analysis of different patients with various cardiac pathologies 7 . Both automatic and manual contour detection are used. The echographic contour detection is performed on four- and two-chamber views of 26 left ventricles and ten left atria as well as on short-axis views of four left ventricles. The MR contour detection is performed on ten four-chamber views and ten short-axis views of left ventricles. Only for this specific validation, the ROI area is not decreased as for indicator dilution applications.

The error of the contour detection, which is referred to as area error, is estimated as the percent area difference between manual and automatic contours. The area is expressed in number of pixels. The error histograms for the echographic and the MR images are shown in Fig. 9. The average error for the echographic images is $10.0 \%$ with a standard deviation equal to $2.0 \%$, while the average error for the MR images is $9.0 \%$ with a standard deviation of $2.2 \%$. An error equal to $8.0 \%$ of the total area is shown in Fig. 12. Fig. 10 shows the correlation between the manual and the automatic area for both the echographic and

${ }^{7}$ All the data are provided by the Departments of Cardiology and Radiology of the Catharina Hospital (Eindhoven, The Netherlands).
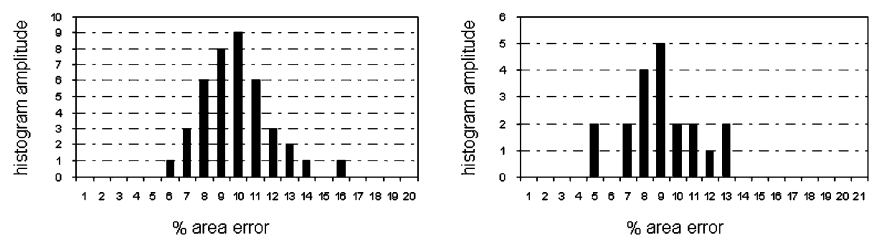

Fig. 9. On the left, the histogram of the percent area error between automatic and manual measurements for the echographic images. On the right, the same histogram for the MR images.
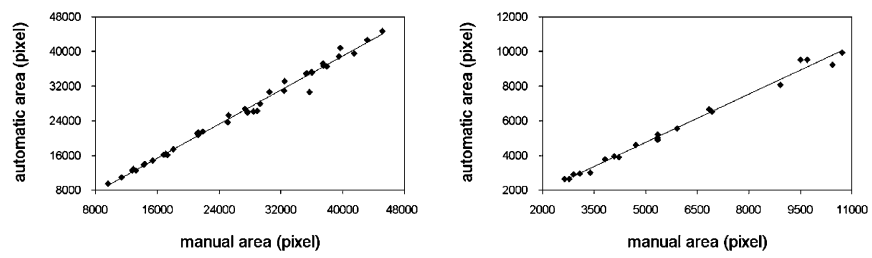

Fig. 10. Automatic versus manual ROI areas (measured in image pixels) for the echographic (on the left) and the MR (on the right) images.
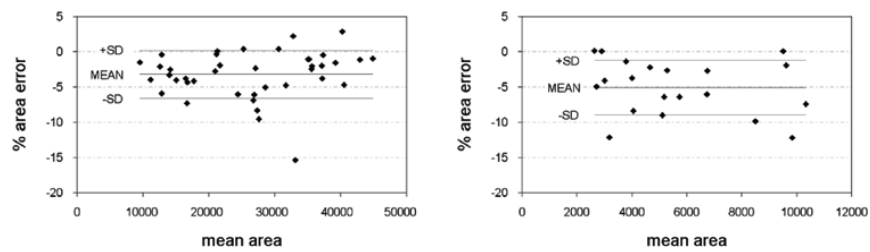

Fig. 11. Bland-Altman plot of the area estimates by automatic and manual contour detection. On the left are the results on echographic images while on the right are the results on MR images. The percent area error is shown together with the mean value (MEAN) and the standard deviation (SD).
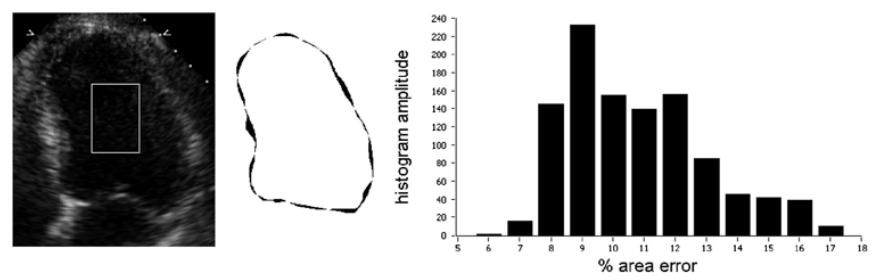

Fig. 12. On the left, the original image with an overlapped rectangle indicating the region were the central point varies to generate the error histogram on the right. The error is represented by the percent area error for different central point positions. In the middle, in black, an $8 \%$ area error is shown.

the MR images. The correlation coefficient is 0.995 and 0.998 , respectively.

The comparison between manual and automatic contour detection is also shown by the Bland-Altman plot in Fig. 11 [60]. The average area error is $-3.2 \%$ (standard deviation equal to $3.4 \%$ ) and $-5.1 \%$ (standard deviation equal to $3.9 \%$ ) for echographic and MR images, respectively. The standard deviation is small and the modest negative bias is a positive characteristic for IDC applications. These results show sufficient accuracy to fulfill the requirements for IDC applications.

All the echographic images are analyzed using the automatic threshold as in (2) except for five images, where the threshold is manually modified by few gray levels $(\leq 5)$. In fact, the automatic threshold in (2) depends on the region where the graylevel standard deviation $\sigma$ is evaluated. Since $\sigma$ is calculated over the complete image, white text or dark regions around echographic images influence the standard deviation. As a result, the automatic threshold may differ from the optimal value 

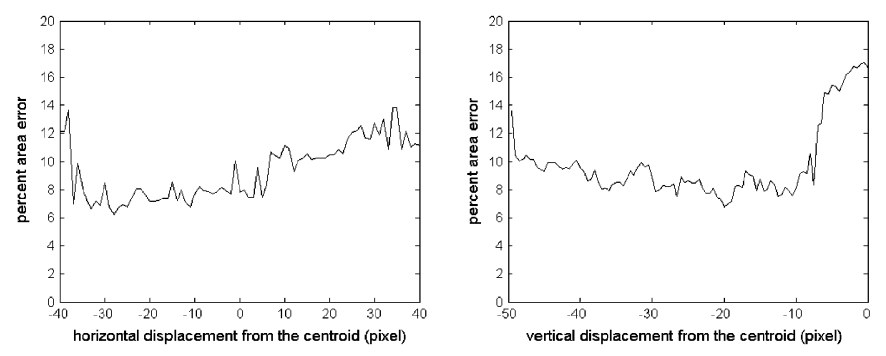

Fig. 13. Percent area error between manual and automatic contours for central point displacements with respect to the manual ROI centroid. The horizontal (on the left) and the vertical (on the right) displacements are calculated along the horizontal and the vertical lines that intercept the contour centroid.
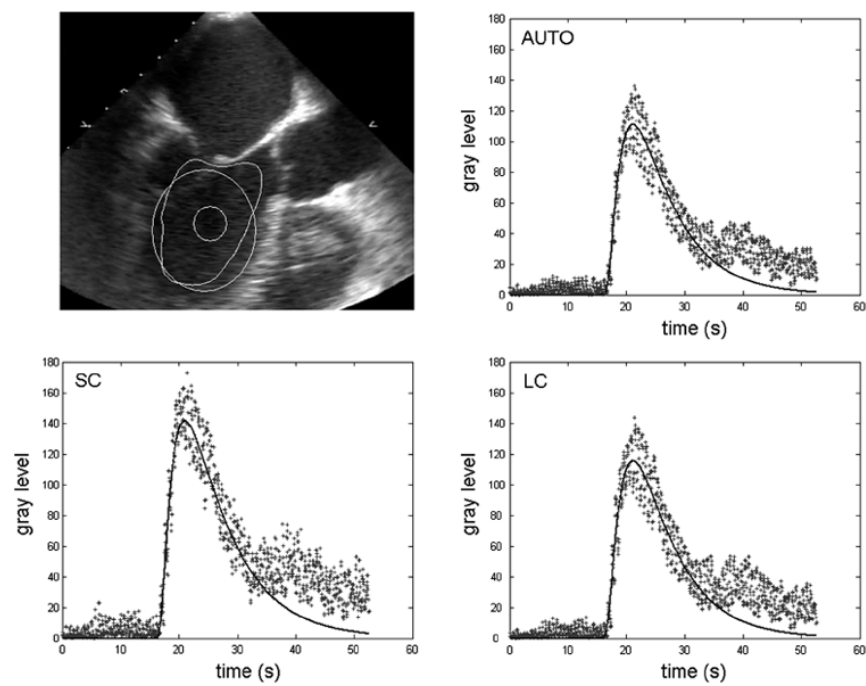

Fig. 14. On the upper left, a mid-esophageal three chamber view is shown together with three different ROIs in the left ventricle. A ROI is defined by automatic detection (AUTO) and two circular ROIs, a large (LC) and a small (SC) circle, are manually placed. The remaining three plots show the measured IDCs from each ROI together with the LDRW model fits.

by few gray levels. Future implementations of the algorithm will include an automatic determination of the region for the evaluation of $\sigma$. The threshold for the analysis of all the MR images, where no text is included, is fixed to 60 .

The sensitivity of the contour detection with respect to the origin of the radial filter is tested to evaluate the reproducibility of the results. The contour of the left ventricle in Fig. 12 is detected for the origin point position varying over the overlapped rectangle. The percent area error histogram is shown in Fig. 12. The error mean value is $9.8 \%$ with a standard deviation equal to $2.3 \%$.

Referring to the same image, Fig. 13 shows the percent area error for vertical and horizontal displacements of the origin point with respect to the contour centroid. We may conclude that for reasonably central positions the algorithm is robust to origin translations.

Fig. 14 shows an application of the algorithm for indicator dilution analysis in humans. A bolus of contrast agent (SonoVue ${ }^{\circledR}$, Bracco Diagnostics, see Appendix A) is injected into a peripheral vein and detected by a trans-esophageal ultrasound transducer in the central circulation. In Fig. 14, a ROI is automatically determined and kept fixed in the LV while the contrast agent is flowing. The average video intensity in the ROI is calculated for each frame and used to build the IDC.

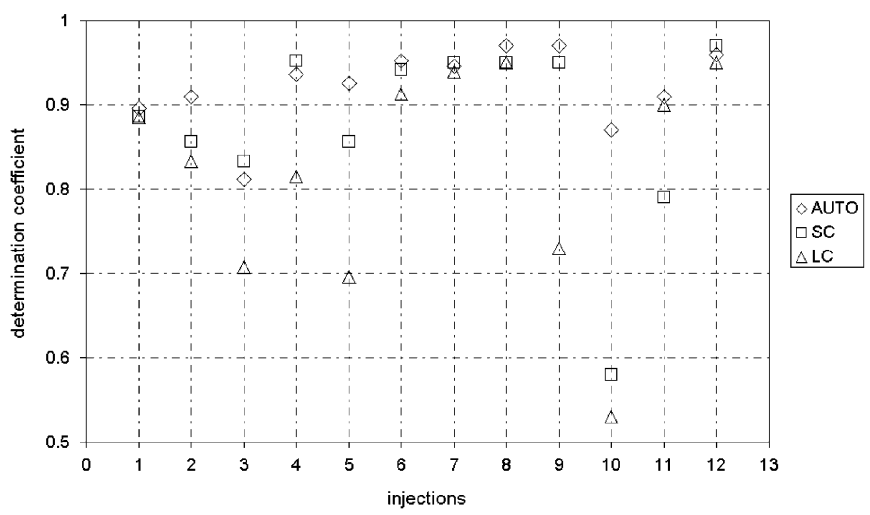

Fig. 15. LDRW-fit determination coefficients for three sets of IDCs, which are measured by the automatic ROI (AUTO), a small circular ROI (SC), and a large circular ROI (LC).

In order to validate the system, the use of the automatic ROI detection is compared to the use of simple manual ROI definitions. Two circular ROIs, a large and a small circle, are added for validation purpose (see Fig. 14). The manual positioning of more complex ROIs is too slow and complex for real-time applications.

The resulting IDCs from the automatic ROI (AUTO), the large circular ROI (LC) and the small circular ROI (SC) are then fitted by a specific model as shown in Fig. 14. The second part of the IDC down-slope (end of the tail) shows the recirculation of the contrast (rise of the contrast concentration with respect of the model fit). Since the cardiac measurements are derived from the first passage IDC, the recirculation introduces an important noise component into the signal. As a consequence, the use of a model becomes necessary to fit the first part of the IDC and estimate the rest of the tail [23].

The local density random walk (LDRW) model (see Appendix B), which was introduced by Sheppard and Savage in 1951 for the IDC interpolation and interpretation [9], [23], [61], [62]-[64] is used to derive the cardiac parameters [23]-[25]. Therefore, the interpolation accuracy is indeed the best parameter to evaluate the contour detection algorithm for IDC measurements. The LDRW fitting is performed by use of a specific fitting algorithm as described in [23].

The same validation as shown in Fig. 14 is performed for 12 trans-esophageal echographic inspections after the injection of a SonoVue ${ }^{\circledR}$ contrast agent bolus. Fig. 15 reports the determination coefficients ( $\rho^{2}$, squared correlation coefficient) of the LDRW fits of the measured IDCs (video-intensity-versus-time curves) for the automatic and the two circular ROIs. The average determination coefficient is 0.922 for the automatic ROI, 0.876 for the small circular ROI, and 0.821 for the large circular ROI (standard deviations equal to $0.041,0.110$, and 0.131 , respectively).

The IDC recorded by the automatic ROI detection shows a more stable behavior and the best average determination coefficient. When a large circle is used, the intrusion of cardiac tissue in the ROI adds large noise components in the IDC. This explains the smallest $\rho^{2}$ for large circular ROIs. Sometimes, the use of a small ROI shows excellent results, slightly better than those obtained by use of automatic ROI detection. However, for low SNR images, the use of a small ROI results in very noisy IDCs (injections 5, 10, and 11 in Fig. 15), and the LDRW-fit shows a much smaller $\rho^{2}$ compared to the automatic ROI detection. 


\section{Discussion AND CONCLUSION}

Due to their accuracy and despite their invasiveness, indicator dilution techniques are the standard techniques for the measurement of CO and PBV. The combined use of ultrasound and UCA allows a noninvasive application of the indicator dilution principles. The simultaneous measurement of all the major cardiac parameters (LV and RV EF, CO, and PBV) could be obtained by a peripheral injection of a single contrast bolus.

The ROI definition is an essential part of the UCA dilution system, since the information contained in the IDC is strongly related to the region of measurement. The available algorithms for contour detection are designed for different purposes, therefore, they do not fulfill all the requirements for the UCA dilution system.

This paper presents a new real-time automatic contour-detection algorithm for indicator dilution applications. It is based on a radial high-pass filter with subsequent correction of the outliers. An initialization with manual contour definition is not needed. The use of different cardiac views is allowed and multiple ROIs can be detected and processed in parallel. Moreover, due to the high flexibility and adaptability to different image features, the algorithm is also applicable to MRI (gadolinium dilutions).

An additional active contour optimization has also been tested. However, for IDC applications, the limited contour accuracy improvement that is obtained with the current implementation does not compensate for the increase of computation time. Future research will include the evaluation of alternative algorithms and implementations for an active contour optimization.

The proposed contour detection algorithm is controlled by several parameters. In particular, the thresholds for the radial filter and the derivative outlier correction are crucial. However, since both thresholds are applied after a derivative operator (after intensity gradient operator and after radial-distance polar derivative), the threshold dependency on specific image features is limited and the algorithm can be applied to different sets of images without changes. However, an important issue ramains the selection of an appropriate region for the evaluation of the image gray level histogram, which is used for the automatic determination of the high-pass filter threshold. Text or dark regions around echographic images influence the resulting threshold. Future implementations will include an automatic selection of the region that is used for the statistical analysis of the image.

The only interaction with the user concerns the positioning of the radial filter origin in the center of the cavity. Therefore, the algorithm user dependency is tested by origin translations. The contour detection shows robustness to filter origin translations in a large area, which includes any reasonable central position.

A comparison with manual ROI definitions is performed for both echographic and MR images and shows accurate results. Furthermore, in the context of the indicator dilution applications, the "quality" of the measured IDCs is evaluated for manual and automatic ROIs. The results show that automatically determined ROIs lead to better fits and easier interpretation of the IDC. In fact, a manual real-time positioning of a ROI on moving structures (e.g., the cardiac walls) is a difficult procedure. Usually a manual ROI is either too large (increased noise due to wall interference) or too small (leading to an increased noise level by poor statistics). As a consequence, the resulting IDC is more noisy and difficult to interpret.

Obviously, both the manual and the automatic solutions are not robust with respect to image translations. The ultrasound transducer and the patient should remain fixed. In this respect, the MRI allows a more robust application of the system, since the patient is the only source of translation. Also movements due to respiration might represent a noise source. The problem could be solved by extending the evaluation time for the minimumarea ROI to an entire respiratory-cycle period.

When the inner part of the cardiac cavities is very noisy (speckle noise), then the risk of detecting a false internal edge must be considered. To overcome the problem, either the threshold of the radial filter (if manually controlled) or the size of the prior median filter should be increased. The addition of a feedback for inlier detection control might also be investigated.

As this paper focuses on the design and the validation of a contour detection algorithm for indicator dilution use, future work will provide results on the cardiac parameter assessments.

\section{APPENDIX}

\section{A. Ultrasound Contrast Agents}

Ultrasound contrast agents are made of a solution of micro-bubbles. The bubbles are composed of air, $\mathrm{SF}_{6}, C_{3} F_{8}$, or other perfluorocarbons encapsuled in a phospholipid, albumin, or polymer shell. SonoVue ${ }^{\circledR}$ is a sulfurhexafluoride bubble encapsuled in a phosholipidic shell. The diameter varies from 0.7 $\mu \mathrm{m}$ to $10 \mu \mathrm{m}$, with an average value equal to $0.25 \mu \mathrm{m}$ [19], [20]. $\mathrm{SF}_{6}$ is a large molecule (molecular weight equal to 146) with low solubility in water (Ostwald solubility ${ }^{8}$ equal to 0.0054 ). As a consequence, the molecule diffusion is low and the contrast very stable. In addition, the presence of a shell creates a strain that compensates the Laplace pressure and stabilizes bubbles against dissolution. The video-intensity decay due to bubble dissolution is $0.2 \mathrm{~dB} / \mathrm{min}$ [65], which is not significant within an IDC measurement procedure that lasts for about 1 minute. The presence of a shell also changes the acoustic properties of the contrast. The bubble elasticity increases, resulting in higher acoustic resonance frequencies (about $3 \mathrm{MHz}$ [19]).

\section{B. Local Density Random Walk Model}

The LDRW model assumes a Gaussian spacial distribution of the contrast that translates with the same velocity of the carrier fluid. The variance of the distribution is a linear function of time. When a contrast bolus is injected and detected in a different site of a hydrodynamic system, then the detected concentration is modeled as given in (3), where $m$ is the injected mass of contrast, $q$ is the volumetric flow, $\lambda$ is a parameter related to the diffusion constant of the system, and $\mu$ is the average time that the contrast takes to go from the injection to the detection site (MTT) [10], [23], [62], [63]

$$
C(t)=\frac{m}{q} e^{\lambda} \sqrt{\frac{\lambda}{2 \pi \mu t}} e^{-\frac{\lambda}{2}\left(\frac{t}{\mu}+\frac{\mu}{t}\right) .}
$$

${ }^{8}$ Volume of gas dissolved per unit volume of solvent. 
The model is solution of the diffusion equation, therefore, it gives a physical interpretation of the dilution process [10]. Furthermore, it shows the best least squares estimation of the IDC when applied to dye-dilution and thermodilution measurements [62]-[64].

\section{ACKNOWLEDGMENT}

The authors like to thank the Departments of Cardiology and Radiology of the Catharina Hospital Eindhoven and in particular A. Jansen and H. van de Bosch for their support and contribution to this research.

\section{REFERENCES}

[1] P. Ask and P. A. Oberg, Blood Flow Measurements. Boa Raton, FL: CRC, 2000.

[2] The Biomedical Engineering Handbook, 2nd ed., J. D. Bronzino, Ed., CRC, Boca Raton, FL, 2000.

[3] R. G. Aarnink, Volume Measurements. Boca Raton, FL: CRC, 2000.

[4] J. R. C. Jansen, "The thermodilution method for clinical assesment of cardiac output," Intensive Care Med., vol. 21, pp. 691-697, 1995.

[5] K. Zierler, "Indicator dilution methods for measuring blood flow, volume, and other properties of biological systems: A brief history and memoir," Ann. Biomed. Eng., vol. 28, pp. 836-848, 2000.

[6] T. Kurita, K. Morita, S. Kato, H. Kawasaki, M. Kikura, T. Kazama, and K. Ikeda, "Lithium dilution cardiac output measurements using a peripheral injection site: Comparison with central injection technique and thermodilution," J. Clin. Monitor. Computing, vol. 15, no. 5, pp. 279-285, 1999.

[7] M. Jonas, D. Hett, and J. Morgan, "Real timecontinuous monitoring of cardiac output and oxygen delivery," Int. J. Intensive Care, vol. 9, no. 1, 2002.

[8] G. Williams, M. Grounds, and A. Rhodes, "Pulmonary artery catheter," Curr. Opinion Intensive Care, vol. 8, pp. 251-256, 2002.

[9] C. W. Sheppard, Basic Principles of Tracer Methods: Introduction to Mathematical Tracer Kinetics. New York: Wiley, 1962.

[10] K. H. Norwich, Molecular Dynamics in Biosystems. New York: Pergamon, 1977.

[11] S. G. Sakka, C. C. Ruhl, U. J. Pfeiffer, R. Beale, A. McLuckie, K. Reinhart, and A. Meier-Hellmann, "Assessment of cardiac preload and extravascular lung water by single transpulmonary thermodilution," Intensive Care Med., vol. 26, pp. 180-187, 2000.

[12] W. Buhre, S. Kazmaier, H. Sonntag, and A. Weiland, "Changes in cardiac output and intrathoracic blood volume: A mathematical coupling of data?," Acta Anaesthesiol Scand, vol. 45, pp. 863-867, 2001.

[13] W. Buhre, A. Weyland, K. Buhre, S. Kazmaier, K. Mursch, M. Schmidt, M. Sydow, and H. Sonntag, "Effects of the sitting position on the distribution of blood volume in patients undergoing neurosurgical procedures," British J. Anaestesia, vol. 84, no. 3, pp. 354-357, 2000.

[14] Handbook of Contrast Echography, Springer-Verlag, Berlin, Germany, 2000. H. Becher, P. N. Burns.

[15] A. Bouakaz, N. de Jong, L. Gerfault, and C. Cachard, "In vitro standard acoustic parameters of ultrasound contrast agents: Definitions and calculations," in Proc. IEEE Ultrasonics Symp., vol. 2, 1996, pp. 1445-1448.

[16] A. Bouakaz, N. de Jong, and C. Cachard, "Standard properties of ultrasound contrast agents," Ultrasound Med. Biol., vol. 24, no. 3, pp. 469-472, 1998.

[17] N. Sponheim, L. Hoff, A. Waaler, B. Muan, H. Morris, S. Holm, M. Myrum, N. de Jong, and T. Skotland, "Albunex-A new ultrasound contrast agent," in Proc. Inst. Elect. Eng. Conf. Acoustinc Sensing and Imaging, Mar. 29-30, 1993, pp. 103-107.

[18] B. Herman, S. Einav, and Z. Vered, "Feasibility of mitral flow assessment by echo-contrast ultrasound, part I: Determination of the properties of echo-contrast agents," Ultrasound Med. Biol., vol. 26, no. 5, pp. 785-795, 2000.

[19] M. Shneider, "Characteristics of SonoVue," Echocardiography, pt. 2, vol. 16, no. 7, pp. 743-746, 1999.

[20] J. M. Gorce, M. Arditi, and M. Schneider, "Influence of bubble size distribution on echogenecity of ultrasound contrast agents: A study of SonoVue," Invest. Radiol., vol. 35, no. 11, pp. 661-671, 2000.

[21] W. R. Hedrick, D. L. Hykes, and D. E. Starchman, Ultrasound Physics and Instrumentation, 3rd ed. St. Louis, MO: Mosby, 1995.
[22] V. Uhlendorf, "Physics of ultrasound contrast imaging: Scattering in the linear range," IEEE Trans. Ultrason., Ferroelect. Freq. Control, vol. 41, no. 1, pp. 70-79, Jan. 1994.

[23] M. Mischi, A. A. C. M. Kalker, and H. H. M. Korsten, "Videodensitometric methods for cardiac output measurements," EURASIP J. Appl. Signal Process., vol. 2003, no. 5, pp. 479-489, 2003.

[24] M. Mischi, A. H. M. Jansen, A. A. C. M. Kalker, and H. H. M. Korsten, "Intra-thoracic blood volume assessment by dilution of ultrasound contrast agents," in Proc. IEEE-UFFC Int. Ultrasonics Symp., Honolulu, HI, Oct. 5-8, 2003, pp. 1179-1182.

[25] M. Mischi, A. A. C. M. Kalker, and H. H. M. Korsten, "Blood volume measurements by videodensitometric analysis of ultrasound-contrastagent dilution curves," in Proc. IEEE-EMBS 25th Annu. Int. Conf. IEEE Engineering in Medicine and Biology Society, Cancun, Mexico, Sept. 17-21, 2003, pp. 791-794.

[26] C. S. Sehgal and P. H. Arger, "Mathematical modeling of dilution curves for ultrasonographic contrast," J. Ultrasound Med., vol. 16, pp. 471-479, 1997.

[27] D. Rovani, S. E. Nissen, E. Jonathan, M. Smith, A. L'Abbate, O. L. Kuan, and A. N. De Maria, "Contrast echo washout curves from left ventricle: Application of basic principles of indicator-dilution theory and calculation of ejection fraction," J. Am. Coll. Cardiol., vol. 10, pp. $125-134,1987$.

[28] L. Gerfault, E. Helms, V. Bailleau, N. Rognin, G. Finet, M. Janier, and C. Cachard, "Assessing blood flow in isolated pig heart with USCA," in Proc. IEEE Ultrasonics Symp., vol. 2, 1999, pp. 1725-1728.

[29] T. Gustavsson and M. Beckman-Suurkula, "Videodensitometric measurements in contrast-echocardiography," Comput. Cardiol., pp. 709-712, 1996.

[30] H. Bleeker, K. Shung, and J. Barnhart, "On the application of ultrasonic contrast agents for blood flowmetry and assesment of cardiac perfusion," J. Ultrasound Med., vol. 9, pp. 461-471, 1990.

[31] P. A. Heidenreich, J. G. Wiencek, J. G. Zaroff, S. Aroson, L. J. Segil, P. V. Haper, and S. B. Feinsten, "In vitro calculation of flow by use of contrast ultrasonography," J. Am. Soc. Echocardiogr., vol. 6, pp. 51-61, 1993.

[32] X. Chen, K. Q. Schwarz, D. Phillips, S. D. Steinmetz, and R. Schlief, "A mathematical model for the assessment of hemodynamic parameters using quantitative contrast echocardiography," IEEE Trans. Biomed. Eng., vol. 45, no. 6, Jun. 1998.

[33] D. Rovai, M. Lombardi, L. Taddei, A. Mazzarisi, L. Landini, G. Ghelardini, A. Distane, A. Benassi, and A. L'Abbate, "Flow quantitation by contrast echocardiography," Int. J. Cardiac Imag., vol. 9, pp. 21-27, 1993.

[34] D. Rovai, M. Lombardi, A. Mazzarisi, L. Landini, L. Taddei, A. Distane, A. Benassi, and A. L'Abbate, "Flow quantitation by radio frequency analysis of contrast echocardiography," Int. J. Cardiac Imag., vol. 9, pp. 7-19, 1993.

[35] L. J. Bos, J. J. Piek, and J. A. E. Spaan, "Effects of shadowing on the time-intensity curves in contrast echocardiography: A phantom study," Ultrasound Med. Biol., vol. 22, no. 2, pp. 217-227, 1996.

[36] M. Mischi, A. H. M. Jansen, A. A. C. M. Kalker, and H. H. M. Korsten, "A new ultrasound dilution method for EF quantification," presented at the 15th Annu. Scientific Sessions of the American Society of Echocardiography, San Diego, CA, June 26-30, 2004.

[37] H. Feigenbaum, Echocardiography, 5th ed. Baltimore, MD: William \& Wilkins, 1994, ISBN 0-8121-1692-5.

[38] A. P. Dhawan, Medical Image Analysis. Piscataway, NJ: IEEE Press, 2003.

[39] A. K. Jain, Fundamentals of Digital Image Processing. Upper Saddle River: Prentice-Hall, 1989.

[40] M. Kass, A. Witkin, and D. Terzopoulos, "Snakes: Active contour models," Int. J. Comput. Vis., vol. 1, no. 4, pp. 321-331, 1987.

[41] N. Otsu, "A threshold selection method from gray-level histograms," IEEE Trans. Syst., Man, Cyben., vol. SMC-9, no. 1, pp. 62-66, Jan. 1979.

[42] A. D. Parker, A. Hill, C. J. Taylor, T. F. Cootes, X. Y. Jin, and D. G Gibson, "Application of point distribution models to automated analysis of echocardiograms," Comput. Cardiol., 1994.

[43] A. Lantis, C. J. Taylor, and T. F. Cootes, "Automatic interpretation and coding of face images using flexible models," IEEE Trans. Patterns Anal. Machine Intell., vol. 19, no. 7, pp. 743-756, Jul. 1997.

[44] B. van Ginneken, A. F. Frangi, J. J. Staal, B. M. ter Haar Romeny, and M. A. Viergever, "Active shape model segmentation with optimal features," IEEE Trans. Med. Imag., vol. 21, no. 8, Aug. 2002.

[45] R. G. Aarnink, "Automated Contour Detection, Design, and Clinical Applications in Urology," Ph.D. thesis, Catholic Univ. Nijmegen, Sep. 10, 1996. 
[46] T. Fujino et al., "New method of on-line quantification of regional wall motion with automated segmental motion analysis," J. Am. Soc. Echocardiogr., vol. 14, no. 9, pp. 892-901, 2001.

[47] H. G. Bosch, S. C. Mitchell, B. P. F. Lelieveldt, F. Nijland, O. Kamp, M. Sonka, and J. H. C. Reiber, "Active appearance-motion models for fully automated endocardial contour detection in time sequences of echocardiograms," in Proc. CARS-CVI, Berlin, Germany, June 27-30, 2001.

[48] I. Craw, N. Costen, T. Kato, and S. Akamatsu, "How should we represent faces for automatic recognition?," IEEE Trans. Patterns Anal. Machine Intell., vol. 21, no. 8, pp. 725-736, Aug. 1999.

[49] T. F. Cootes, G. J. Gareth, and C. J. Taylor, "Active appearance models," IEEE Trans. Patterns Anal. Machine Intell., vol. 23, no. 6, pp. 681-685, Jun. 2001.

[50] T. F. Cootes and C. J. Taylor, "Constrained active appearance models," in Proc. IEEE 8th Int. Conf.Computer Vision, vol. 1, Vancouver, BC, Canada, July 7-14, 2001, pp. 748-754.

[51] K. Althoff, G. Hamarneh, M. C. Suurkula, and T. Gustavsson, "Tracking contrast in echography by a combined snake and optical flow technique," Comput. Cardiol., vol. 27, pp. 29-32, 2000.

[52] S. Osher and J. Sethiam, "Fronts propagating with curvature-dependent speed: Algorithms based on Hamilton-Jacobi formulation," J. Computational Phys., vol. 79, pp. 12-49, 1988

[53] N. de Jong, A. Bouakaz, and F. J. T. Cate, "Contrast harmonic imaging," Ultrasonics, vol. 40, pp. 567-573, 2002.

[54] D. E. Goertz, S. W. S. Wong, C. T. Chin, E. Cherin, P. N. Burns, and F. S. Foster, "Non-linear scattering from microbubbles contrast agents in the 14-40 MHz range," in Proc. IEEE Ultrason. Symp., Atlanta, GA, 2001, pp. $1747-1750$.

[55] J. Morkenborg, M. Pedersen, F. T. Jensen, H. Stodkilde-Jorgensen, J. C Djurhuus, and J. Frokiaer, "Quantitative assessment of Gd-DTPA contrast agent from signal enhancement: An in-vitro study," Magn. Reson. Imag., vol. 21, pp. 637-643, 2003.

[56] F. Wittlich, K. Koho, G. Mies, D. Norris, and M. Hoehn-Berlage, "Quantitative measurement of regional blood flow with gadolinium diethylenetri-aminepentaacetate bolus track NMR imaging in cerebral infarcts in rats: Validation with the iodio $\left[{ }^{14} \mathrm{C}\right]$ antipyrine technique," Proc. Nat. Acad. Sci., vol. 92, pp. 1846-1850, 1995.

[57] N. Frieland and D. Adam, "Automatic ventricular cavity boundary detection from sequential ultrasound images using simulated annealing," IEEE Trans. Med. Imag., vol. 8, no. 4, Dec. 1989.

[58] A. A. Amini, T. E. Weymouth, and R. C. Jain, "Using dynamic programming for solving variational problems in vision," IEEE Trans. Patterns Anal. Machine Intell., vol. 12, no. 9, pp. 855-867, Sep. 1990.

[59] . Pattern Recognition and Image Processing Group, Vienna University of Technology. [Online]. Available: http://www.prip.tuwien.ac.at/Research/3DVision/Cameras/tutorial.html

[60] J. M. Bland and D. G. Altman, "Statistical methods for assessing agreement between two methods of clinical measurement," Lancet, vol. 1, pp. 307-310, 1986

[61] C. W. Sheppard and L. J. Savage, "The random walk problem in relation to the physiology of circulatory mixing," Phys. Rev., vol. 83, pp. 489-490, 1951.

[62] M. E. Wise, "Tracer dilution curves in cardiology and random walk and lognormal distributions," Acta Phisiol. Pharmacol. Neerl., vol. 14, pp. $175-204,1966$.

[63] J. M. Bogaard, S. J. Smith, A. Versprille, M. E. Wise, and F. Hagemeijer, "Physiological interpretation of skewness of indicator-dilution curves; Theoretical considerations and pratical application," Basic. Res. Cardiol., vol. 79, pp. 479-493, 1984.

[64] E. A. Reth and J. M. Bogaard, "Comparison of a two-compartment model and distributed models for indicator dilution studies," Med. Biol. Eng. Comput., vol. 21, pp. 453-459, 1983.

[65] R. J. Eckersley, C. A. Sennoga, R. C. Campbell, and M. J. K. Blomley, "Characterization of novel microbubble contrast agents," presented at the IEEE-UFFC Int. Ultrasonics Symp., Honolulu, HI, Oct. 5-8, 2003.

[66] P. J. A. Frinking and N. de Jong, "Acousrtic modeling of shell-encapsuled gas bubbles," Ultrasound Med. Biol., vol. 24, no. 4, pp. 523-533, 1998.

[67] N. de Jong, L. Hoff, T. Skotland, and N. Bom, "Absorption and scatter of encapsuled gas filled microsphere: Theoretical considerations and some measurements," Ultrasonics, vol. 30, no. 2, pp. 95-103, 1992.
[68] C. T. Chin, M. Versluis, C. Lancee, and N. de Jong, "Free oscillation of microbubbles as observed using a new 25 million frames per second camera," presented at the IEEE-UFFC Int. Ultrasonics Symp., Honolulu, HI, Oct. 5-8, 2003.

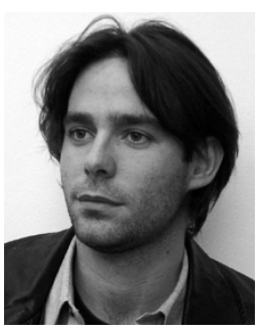

Massimo Mischi (S'04-M'04) was born in Rome, Italy, in 1973. In 1999, he received the M.S. degree in electronic engineering at La Sapienza University of Rome. In his final thesis he designed and validated a new device for optical-performance measurements of clinical fiber-optic endoscopes. In 2002 he completed a two-year Post-Masters program in Technological Design, Information, and Communication Technology, at the Eindhoven University of Technology, Eindhoven, The Netherlands, studying on the application of indicator dilution theory and ultrasound contrast agents for cardiac output measurements. Currently, he is a $\mathrm{Ph}$.D. degree candidate and a research assistant at the Eindhoven University of Technology.

His research concerns the quantification of cardiac parameters by means of contrast echocardiography.

Mr. Mischi is registered in the Italian Public Register of Engineers and in the Dutch Royal Institute of Designers.

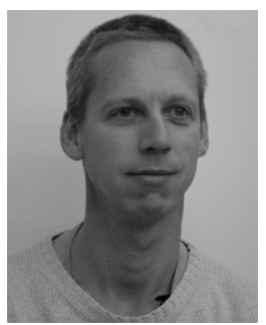

Antonius A. C. M. Kalker (M'93-SM'99-F01) was born in The Netherlands in 1956. He received the M.S. and Ph.D. degrees in mathematics in 1979 from the University of Leiden, Leiden, The Netherlands, in 1979 and 1986, respectively.

From 1979 until 1983, while he was a Ph.D. degree candidate, he worked as a Research Assistant at the University of Leiden. From 1983 until December 1985, he worked as a Lecturer at the Computer Science Department of the Technical University of Delft, Delft, The Netherlands. In December 1985, he joined the Philips Research Laboratories, Eindhoven. Until January 1990, he worked in the field of computer-aided design. He specialized in (semi-) automatic tools for system verification. Currently, he is a member of the Digital Signal Processing group of Philips Research. His research interests include wavelets, multirate signal processing, motion estimation, psycho-physics, digital video compression, medical imaging, digital watermarking, and multimedia security.

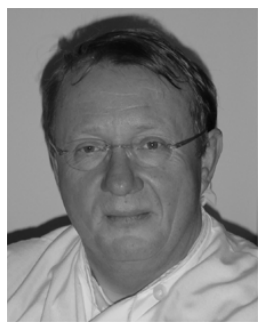

Henricus H. M. Korsten was born in the Netherlands in 1953. He studied medicine in Groningen and graduated as a medical doctor in 1978. During his training as an anesthesiologist in Utrecht, he started Ph.D. degree study on the measurement of intrathoracic fluid content during open-heart surgery. In November 1984, he received the Ph.D. degree in medicine form the University of Leiden, Leiden, The Netherlands.

Since June 1982, he is a Staff Member of the Department of Anesthesiology, Intensive Care and PainTreatments in the Catharina Hospital, Eindhoven. From 1989 until 1993, he was the Chairman of the professional medical staff of this hospital. He is also an Intensivist and was head of the Intensive-Care during the period 1993 until 2001. During this period he was one of the initiators of a national intensive-care database. He was also involved in research-projects on data-storage and data-mining of patient-data, as well as the development of artificial intelligence in the intensive-care. In 2001, he was appointed Professor in the Department of Electrical Engineering, Eindhoven University of Technology. 